
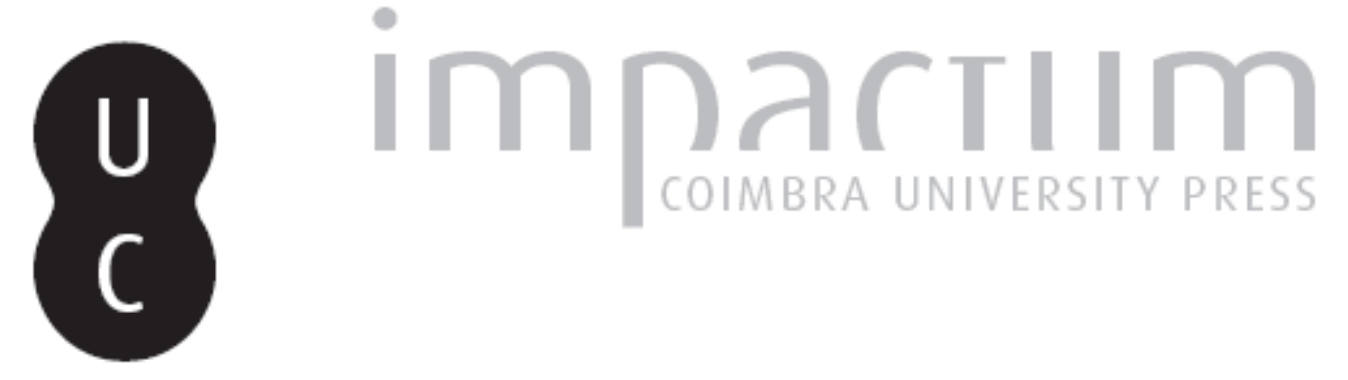

\title{
O mercado de crédito especializado ao consumo em Portugal
}

Autor(es): $\quad$ Garcia, Teresa; Vieira, Paulo

Publicado por: Imprensa da Universidade de Coimbra

URL persistente:

URl:http://hdl.handle.net/10316.2/43510

DOI:

DOI:https://doi.org/10.14195/2183-203X_45_4

Accessed : $\quad$ 26-Apr-2023 13:59:22

A navegação consulta e descarregamento dos títulos inseridos nas Bibliotecas Digitais UC Digitalis, UC Pombalina e UC Impactum, pressupõem a aceitação plena e sem reservas dos Termos e Condições de Uso destas Bibliotecas Digitais, disponíveis em https://digitalis.uc.pt/pt-pt/termos.

Conforme exposto nos referidos Termos e Condições de Uso, o descarregamento de títulos de acesso restrito requer uma licença válida de autorização devendo o utilizador aceder ao(s) documento(s) a partir de um endereço de IP da instituição detentora da supramencionada licença.

Ao utilizador é apenas permitido o descarregamento para uso pessoal, pelo que o emprego do(s) título(s) descarregado(s) para outro fim, designadamente comercial, carece de autorização do respetivo autor ou editor da obra.

Na medida em que todas as obras da UC Digitalis se encontram protegidas pelo Código do Direito de Autor e Direitos Conexos e demais legislação aplicável, toda a cópia, parcial ou total, deste documento, nos casos em que é legalmente admitida, deverá conter ou fazer-se acompanhar por este aviso.

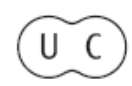




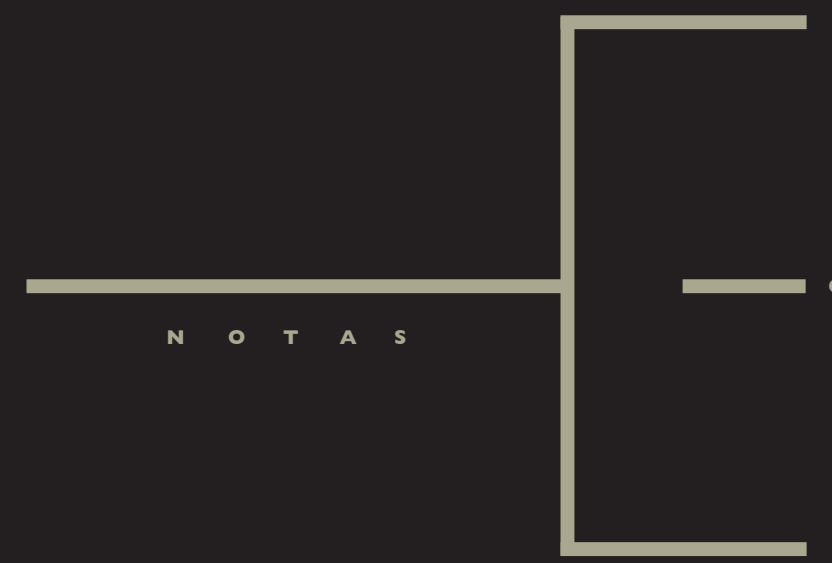

Helder Sebastião / António Portugal Duarte / Gabriel Guerreiro Where is the Information on USD/Bitcoin Hourly Prices?

ANA Fontoura GOUVEIA The Political Economy of Pension Systems with Low-Skilled Labor Mobility:A Cross-Country Analysis

Manuela Magalhães / Ana Paula Africano Panel Analysis of the FDI Impact on International Trade Revisited

Teresa Garcia / Paulo Vieira

O Mercado de Crédito Especializado ao Consumo em Portugal

JoÃo SOUSA ANDRADE As Reformas Monetárias e o Euro 


\title{
O Mercado de Grédito Espegializado ao Gonsumo em Portugal
}

\author{
Teresa Garcia \\ Paulo Vieira
}

Received for publication: February 27, 2017

Revision accepted for publication: May 17, 2017

\section{RESUMO}

O artigo analisa o mercado de crédito ao consumo em Portugal no período 1998-2011. A análise econométrica considera diversos modelos de regressão linear para identificar e avaliar o impacto das variáveis que o determinam, assente na teoria do ciclo de vida do consumo, na economia do crédito ao consumo e na teoria das finanças pessoais. Os resultados da estimação dos modelos permitiram concluir que as variações trimestrais do crédito concedido num dado trimestre são fortemente influenciadas pela variação do trimestre imediatamente anterior, bem como pelo fator sazonal presente no quarto trimestre de cada ano do período em análise. Adicionalmente, as importações desempenham um papel determinante na evolução da concessão deste tipo de crédito.

\begin{abstract}
This paper analyses the consumer credit market in Portugal for the period from 1998 to 2011. The econometric analysis considers several linear regression models that are used to identify and evaluate the impact of independent variables on consumer credit, based on the life-cycle theory of consumption, the economics of consumer credit, and the theory of household and personal finance. The findings indicate that quarterly variations of consumer credit are significantly influenced by the credit variation of the previous quarter, and, additionally just during the fourth quarter, also by the seasonal factor, for every year under analysis. Furthermore, imports also make a relevant contribution to the evolution of the consumer credit market.

Keywords: Consuming credit; Portugal; descriptive data analysis, linear regression models.
\end{abstract}

JEL Classification: D 12, D 14

Agradecimento: Os autores agradecem os comentários e sugestões do referee anónimo que muito contribuíram para melhorar este artigo, assumindo, naturalmente, a responsabilidade por erros e omissões que permaneçam. Agradece-se ainda o apoio da Fundação para a Ciência e a Tecnologia, sendo este artigo parte do Projeto Estratégico (UID/ECO/00436/2013). 


\section{INTRODUÇÃo}

A liberalização do crédito, em geral, e do crédito ao consumo, em particular, que teve início no final do século xx, em Portugal, conduziu ao seu crescimento significativo. Tornase assim premente analisar a evolução do crédito ao consumo para o período de 1998 a 2011. De facto, em 1998 regista-se a terceira fase da UEM e em 2011 tem lugar uma ajuda externa a Portugal protagonizada pelo FMI, a Comissão Europeia e o BCE (BIS, 2011), o que justifica o período escolhido.

Em Portugal, o tema não tem sido objecto de estudo, embora as preocupações com o sobreendividamento e com a formação financeira tenham conduzido ao Relatório do Inquérito à Literacia Financeira da População Portuguesa (BdP, 2011) e ao Plano Nacional de Formação Financeira (PNFF), lançado em 2011 pelo Conselho Nacional de Supervisores Financeiros (GNSF), visando contribuir para elevar o nível de conhecimentos financeiros da população e promover a adoção de atitudes e comportamentos financeiros adequados (BdP, CMVM, e ISP, 2012; BdP, CMVM, e ASF, 2016). Consequentemente, este artigo tem como objectivo colmatar essa lacura, quer através de uma análise descritiva do mercado de crédito ao consumo quer através de uma análise econométrica que ponha em evidência os factores que determinam a sua evolução.

$\mathrm{O}$ crédito ao consumo enquadra-se na temática do consumo que visa perceber o que motiva os indivíduos a consumir e de que forma estes se encontram restringidos ou influenciados por certos factores, como o rendimento disponível ou a idade (Bertola et al. 2006). Em termos agregados, revê-se o conceito de função consumo e as teorias explicativas do consumo para fundamentar as variáveis explicativas do crédito ao consumo que serão testadas num modelo de regressão linear.

Este trabalho encontra-se dividido em seis secções. Na segunda, é elaborada uma revisão de literatura sobre o consumo e sobre o crédito ao consumo. Na secção 3 apresenta-se a associação representativa das instituições vocacionadas para o financiamento especializado ao consumo em Portugal (ASFAG) e é feita uma breve análise descritiva da série de dados relativos à concessão de crédito ao consumo entre 1998 e 2011. A secção 4 é constituída pelas hipóteses a testar e pelo modelo utilizado. Os resultados são analisados na secção 5 . Por último, na seccão 6, são apresentadas as principais conclusões.

\section{REVisão de Literatura}

A análise do consumo e das decisões de consumo tem evoluído ao longo do tempo, pondo em destaque variáveis com o rendimento disponível, a idade, a dimensão da família, a riqueza, a educação e o acesso ao crédito. Já a temática do crédito ao consumo tem sido pouco estudada.

No início da década de 1950, o modelo prevalecente da função consumo inspirou-se na lei psicológica fundamental do economista John Maynard Keynes, segundo a qual quando o rendimento disponível aumenta, o consumo aumenta numa menor proporção (Attanasio e Weber, 2010).

Devido a algumas inconsistências a nível empírico, foram desenvolvidos, na mesma década, o modelo do ciclo de vida por Modigliani e Brumberg (1954) e o modelo do 
rendimento permanente por Friedman (1957). A hipótese do ciclo de vida estabelece que a principal razão para se poupar é a necessidade de precaver o futuro em termos de rendimento, nomeadamente quando se atinge uma idade avançada (Modigliani, 1988; Deaton, 2005). Não obstante, Banks et al. (1998) referem que a diferença entre a descida no consumo expectável e e a descida no consumo real resulta de choques inesperados, nomeadamente ao nível da informação disponível perto da idade da reforma, em oposição a uma falta de racionalidade no padrão de consumo ao longo do ciclo de vida (Dilnot et al., 1994).

A teoria do rendimento permanente assenta no pressuposto de que os indivíduos procuram manter o seu nível de consumo estável, evitando que este oscile como resultado de variações do rendimento disponível a curto prazo (Meghir, 2004). Assim, os indivíduos estabelecem o seu consumo com base numa perspectiva de rendimento de longo prazo, ou seja, de rendimento permanente, que se obtém como uma média do rendimento recebido pelo indivíduo ao longo da sua vida.

O aspecto mais relevante de ambos os modelos é o de tratar as decisões de consumo como parte de um problema de alocação intertemporal, sendo o horizonte temporal a principal diferença entre eles nas suas formulações originais. Finito no primeiro e infinito no segundo (Attanasio, 1999; Browning e Crossley, 2001).

Bertola et al. (2006) consideram que as famílias adoptam o seu nível de consumo óptimo em cada período sujeito a uma restrição orçamental intertemporal, de forma a controlar a sua volatilidade. Assim, a poupança, como refere Courdet (1990), responde somente a dois motivos: o reembolso de dívidas realizadas no início da vida activa, e a constituição de um capital que permita financiar a reforma. Deste modo, grande parte da riqueza é gerada pela acumulação para ser consumida no fim da vida e não por via das transferências intergeracionais. Masson (1991) refere que a hipótese do ciclo de vida, mais do que tudo o resto, fundamenta um determinado esquema de escolhas ou de decisões patrimoniais dito de "procedimento hierarquizado" com três etapas fundamentais. Em primeiro lugar, a família, cujos rendimentos e necessidades (dimensão, etc.) são por hipótese previamente fixados, efectua a arbitragem intertemporal entre consumo e poupança de acordo com o perfil de acumulação óptimo sobre o horizonte da sua existência. Numa segunda fase, procede à decisão relativa à combinação de classes de activos a deter, dada a poupança segundo a idade. Por último, na terceira etapa, faz as escolhas de investimento em cada activo que, no seio da restrição global imposta pelas escolhas de poupança, respondem melhor às suas necessidades.

O mais relevante deste esquema é que vem ao encontro das preocupações actuais que se manifestam com o surgimento do "conselho patrimonial global" (por contrapartida da gestão corrente da carteira) e com o crescendo de produtos de poupança de longo prazo, assentes na preparação da reforma, mas também dos produtos de crédito ao consumo.

A hipótese do ciclo de vida conduz a diversas possibilidades de análise quando diferentes tipos de comportamento são admitidos para os perfis do rendimento e, por conseguinte, do património. A evidência empírica mostra que os comportamentos patrimoniais se explicam, para uma parte não negligenciável de indivíduos e famílias, pela existência de restrições de liquidez que limitam fortemente as possibilidades de um património 
líquido negativo. As consequências dessas restrições de liquidez, quando aliadas à incerteza quanto ao futuro, são precisamente as ocorrências de heranças "acidentais" e de heranças "voluntárias". Em oposição, a inexistência de restrições de liquidez, através do acesso ao crédito ao consumo sem limite, pode conduzir a endividamento e a património líquido negativo. Esta possibilidade mostra os perigos potenciais associados a um desenvolvimento não controlado do crédito ao consumo e ao sobreendividamento, como acontece já em alguns países (EC, 2008). Por outro lado, poderá justificar a tendência decrescente das taxas de poupança dos particulares em muitos países, incluindo Portugal (Rocher e Stierle, 2015; Garcia, 2013; Costa e Farinha, 2012), com consequências no financiamento das respetivas economias.

Torna-se assim pertinente analisar os factores que determinam o crédito ao consumo. Bertola et al. (2006) afirmam que a concessão de crédito ao consumo enfrenta os mesmos problemas que o crédito empresarial, ao ser afectada pelo risco moral e por problemas de selecção adversa decorrentes da assimetria de informação. A taxa de juro praticada deverá ser tão reduzida quanto possível, de forma a ser atractiva para consumidores com capacidade de cumprimento, bem como para controlar o risco de contraparte dos restantes consumidores. Campbell (2006) refere que algumas famílias cometem sérios erros nas decisões de investimento, devido ao facto do conjunto de produtos financeiros disponível ser confuso e de um elevado grau de complexidade. Neste contexto, a expansão da educação financeira junto das famílias, embora relevante, poderá não ser tão eficaz como a regulamentação do consumo de forma a solucionar os problemas/custos que advêm das más decisões de investimento. A probabilidade das famílias declararem insolvência aumenta com o benefício económico que daí advém (Fay et al., 2002). Alguns estudos, baseados em inquéritos, concluem que os principais factores que levam as famílias a declarar insolvência são problemas de saúde, de desemprego e de divórcio (Sullivan et al., 2000; Himmelstein et al., 2009). O fenómeno das insolvências pode também ser uma consequência das políticas de concessão de crédito. Dick e Lehnert (2010) apontam a liberalização do sector bancário, que teve início na década de 80, o grau de abertura ao exterior, e as alterações tecnológicas na concessão de crédito aos consumidores, como causas significativas da expansão do crédito ao consumo e do aumento exponencial de consumidores a declararem insolvência.

Em Portugal, não existem estudos que analisem os factores que determinam o crédito ao consumo. Não obstante, Santos et al. (2013) oferecem uma revisão crítica de literatura sobre o consumo e o crédito às famílias que procura identificar os principais contributos e desvantagens das abordagens disciplinares para a compreensão dos múltiplos fatores que os influenciam.

O presente artigo pretende pôr em evidência os determinantes do crédito total ao consumo, em termos agregados, utilizando um modelo linear em que as variáveis explicativas escolhidas se baseiam na revisão de literatura mais relevante atrás exposta mas também na análise histórica da variável dependente, mediante um conjunto de cinco hipóteses. Assim, são consideradas como variáveis explicativas o grau de abertura ao exterior, a procura interna, as vendas de automóveis ligeiros de passageiros, as importações, as exportações, e o produto interno bruto. 


\section{Enquadramento da ASFAG e do Grédito}

A ASFAC (Associação de Instituições de Crédito Especializado) ${ }^{1}$ data do ano de 1991 e é a organização representativa do sector do financiamento especializado ao consumo em Portugal. ${ }^{2}$ Dos vários objectivos a que a ASFAC se propõe, destacam-se a defesa e representação dos interesses do sector do crédito especializado perante as entidades de tutela ${ }^{3}$, a representação do sector junto de entidades públicas ${ }^{4}$ e privadas $^{5}$ e ainda a criação e promoção de informação relacionada com o mercado do sector de crédito especializado que possa ser útil para várias entidades do foro público ou privado, e a nível nacional e internacional. Internacionalmente, a ASFAC representa as entidades que a compõe junto da EUROFINAS (European Federation of Finance House Associations) ${ }^{6}$.

É possível analisar o setor através dos dados trimestrais sobre concessão de crédito, fornecidos pela ASFAC, para o período 1998-2011 (Figura 1). Os valores trimestrais médios situam-se próximos dos 1.100 milhões de euros. O primeiro e o quarto trimestre são geralmente os trimestres em que se concede respectivamente o menor e o maior montante de crédito.

O crédito concedido é repartido em quatro tipos principais, Crédito Clássico, Crédito Fornecedores (stock), Crédito Revolving e ainda Outros. ${ }^{7}$ A tendência é globalmente crescente até à Grande Recessão de 2008, data a partir da qual se regista uma maior volatilidade (Figura 2).

${ }^{1}$ Esta entidade tinha como designação Associação de Sociedades Financeiras para Aquisições a Crédito até ao ano 2005. Consultar www.asfac.pt.

${ }^{2}$ Actualmente é composta por 30 membros, dos quais 26 instuições de crédito especializadas no financiamento ao consumo e as restantes prestadoras de serviços essenciais ao funcionamento das anteriores.

3 Nomeadamente o Ministério das Finanças e o Banco de Portugal.

4 Nomeadamente a Direcção Geral do Consumidor e o Instituto Nacional de Estatística (INE).

${ }^{5}$ Nomeadamente a Associação Portuguesa de Bancos (APB) e a Associação de Comércio Automóvel de Portugal (ACAP).

6 A EUROFINAS foi criada em 1959 pelas associações da Áustria, Bélgica, França, Alemanha, Reino Unido, Holanda e Suécia, onde o mercado de crédito especializado já se encontrava bastante desenvolvido.

${ }^{7}$ De acordo com a ASFAC, o Crédito Clássico consiste no financiamento da aquisição de bens ou serviços para o consumidor final cuja liquidação tem um plano de amortização pré-definido. Este tipo de crédito é habitualmente concedido a particulares, empresários em nome individual e empresas e é usado para financiar a aquisição de meios de transporte, equipamentos para o lar ou outro tipo de equipamentos, em concreto autocaravanas, barcos e roulottes. O Crédito Fornecedores (stock) visa permitir a aquisição de bens para posterior revenda, independentemente do sector de actividade retalhista. O Crédito Revolving não está intrinsecamente ligado à aquisição de um bem ou serviço, tal como acontece com o crédito pessoal. Porém, o ponto em que diferem estes dois tipos de crédito é o facto do crédito revolving não ter um prazo fixo para a amortização de dívida. Este assenta num plano de amortização de dívida flexível e num plafond de crédito, Os exemplos mais comuns do crédito revolving são a abertura de crédito em conta corrente e os cartões de crédito. Por último, o Crédito Outros inclui tipos de crédito que não estão definidos nos anteriores. 


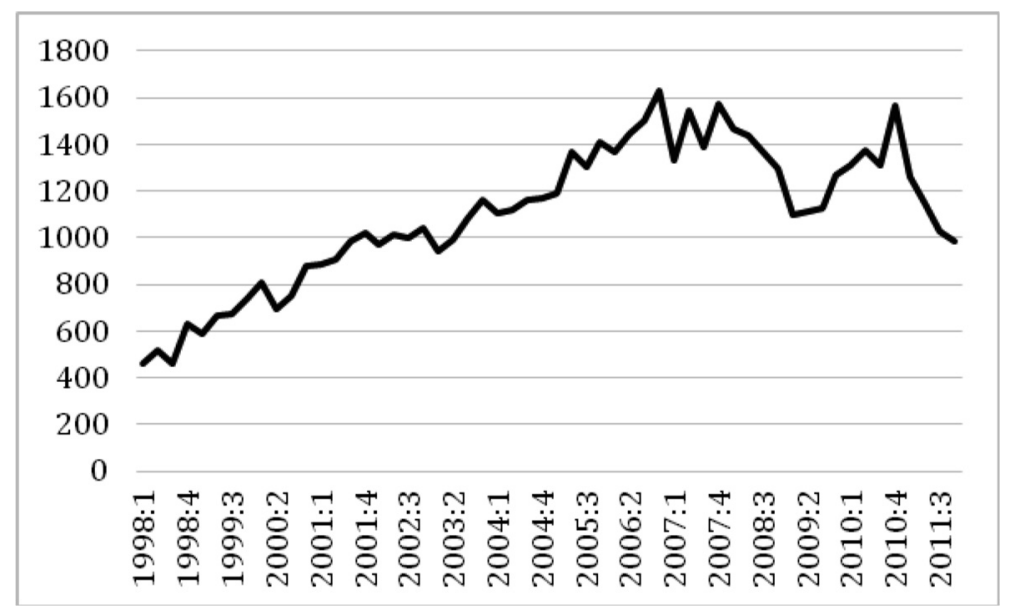

Figura 2: Crédito ao consumo por tipo em Portugal, 1998:1-2011:4 (em milhões de Euros)

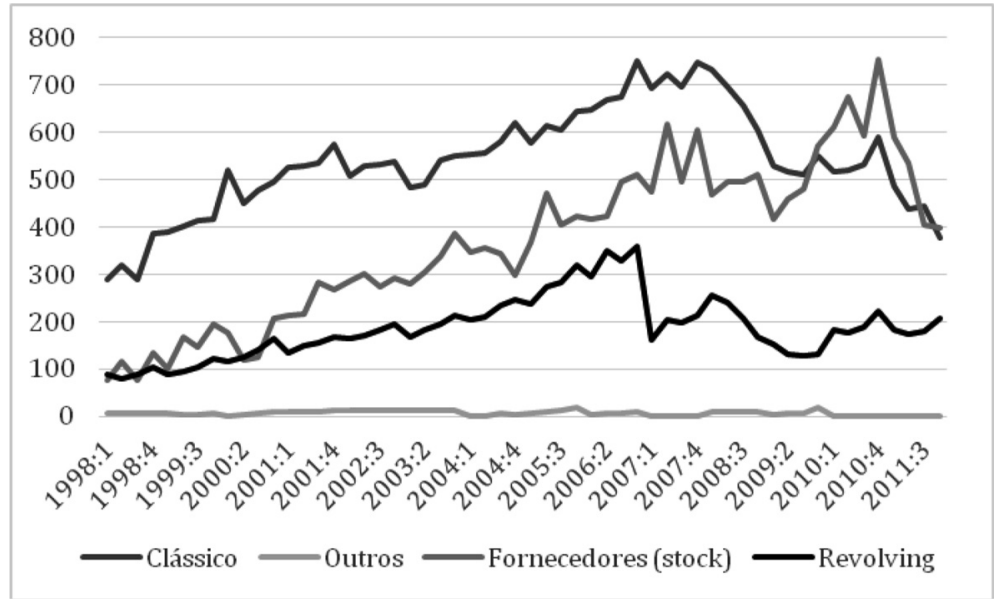

Constata-se que a rubrica Outros não é relevante, o Crédito Clássico representa quase metade do crédito total, seguindo-se o Crédito Fornecedores e o Crédito Revolving. O Crédito Clássico representou em média um volume de cerca de 540 milhões de euros por trimestre e de 2.160 milhões de euros anuais. Este tipo de crédito subdivide-se consoante o tipo de 
cliente, particulares e empresas, e o tipo de produto financiado. O crédito destinado a particulares representa cerca de 90,87\% do Crédito Clássico, estando os restantes 9,13\% destinados às empresas.

O Crédito Fornecedores tem-se afirmado como a segunda maior rubrica do crédito total, revelando que as empresas têm vindo continuamente a recorrer a este tipo de crédito como forma de financiamento. Os dois anos em que se registaram os maiores montantes concedidos, 2007 com um valor de 2.193 milhões de euros e 2010 com um valor de 2.633 milhões de euros, foram também os anos após os quais se registaram as maiores variações negativas, respectivamente $10,08 \%$ e $26,67 \%$, contribuindo assim para um período de instabilidade.

Por último, o Crédito Revolving representou 16,94\% do crédito total concedido, com um valor médio de 746 milhões de euros anuais. Em 2006 foi concedido o maior montante de crédito revolving registado no período, num valor de 1.335 milhões de euros, seguindo-se de uma quebra de $41,73 \%$ no ano de 2007.

Foram celebrados, em média, 508.441 contratos de Crédito Clássico por ano para o período em análise, dos quais 493.022 (96,97\%) destinados a particulares e 15.419 (3,03\%) às empresas. Em termos de distribuição trimestral, o quarto trimestre é aquele que apresenta, em média, um maior número de contratos celebrados, sendo o segundo trimestre aquele que apresenta um menor número de contratos celebrados. O valor médio anual dos contratos de Crédito Clássico situou-se nos 4.249€, valor que é bastante próximo do valor médio por contrato destinado a particulares (3.981€) e distante do destinado às empresas (13.144€). Embora os contratos celebrados com as empresas apresentem valores médios bastante superiores aos dos particulares, estes representam apenas 3,03\% do total dos contratos celebrados.

\section{Hipóteses e Modelo}

Nesta secção apresentam-se as hipóteses testadas relativamente aos factores determinantes do crédito ao consumo e o modelo utilizado.

\subsection{Hipóteses}

Decorrente da análise descritiva do crédito ao consumo, estabeleceram-se diversas hipóteses sobre as variáveis que poderão determinar o crédito ao consumo, que se seguem:

H1: A concessão de crédito ao consumo (CASFAC) é determinada pelo Grau de Abertura ao Exterior (GAE). ${ }^{8}$

H2: A Procura Interna (PI) influencia a concessão de crédito ao consumo. ${ }^{9}$

H3: A concessão de crédito ao consumo é determinada pelas Vendas de Automóveis Ligeiros de Passageiros (VAACAP).

\footnotetext{
${ }^{8}$ G. A. E. $=($ Exportações + Importações $) / P I B$.

9 Procura Interna $=\mathrm{C}+\mathrm{I}_{\mathrm{B}}+\mathrm{G}$, onde $\mathrm{C}$ é o Consumo, $\mathrm{I}_{\mathrm{B}}$ é o Investimento Bruto, e G são os Gastos Públicos.
} 
H4: As Importações (I) e as Exportações (E) influenciam a concessão do crédito ao consumo.

H5: A concessão de crédito ao consumo é influenciada pela riqueza gerada no país dada pelo Produto Interno Bruto (PIB).

\subsection{DAdos}

Como referido anteriormente, os dados relativos à concessão de crédito ao consumo têm frequência trimestral, totalizando 56 observações ao longo do período de 1998 a 2011 , e foram cedidos pela ASFAC. Foi utilizado o deflator para os servicos de intermediação financeira do Eurostat. Não foi efectuado qualquer tipo de ajustamento sazonal prévio a esta série de dados antes de aplicar o deflator a preços constantes de 2005 ajustado sazonalmente.

Em relação às variáveis explicativas consideradas, os dados referentes às variáveis macroeconómicas, nomeadamente o PIB, as Importações, as Exportações e a Procura Interna, foram retirados do Eurostat. Foram extraídos os valores correntes ajustados sazonalmente e aplicados os deflatores específicos para cada variável. Todos os deflatores utilizados foram retirados do portal do Eurostat, encontrando-se a preços constantes de 2005 e ajustados sazonalmente.

Os dados relativos à venda dos veículos ligeiros de passageiros foram fornecidos pela ACAP (Associação Automóvel de Portugal) ${ }^{10}$. Também à série das vendas de automóveis ligeiros de passageiros foi retirado o efeito sazonal, aplicando o modelo multiplicativo de médias móveis através do software Eviews 7.

\subsection{Modelo}

Foi utilizado um modelo de regressão linear com a seguinte forma geral $y_{t}=\alpha+\beta x_{t}$ $+\gamma y_{t-j}+\delta d_{t}+u_{t}$. A variável dependente, $y_{t}$, representa o crédito ao consumo. $\mathrm{O}$ termo constante da regressão é representado por $\alpha$. Os parâmetros $\beta, \gamma$ e $\delta$, captam a magnitude e o sinal das variáveis independentes $x_{t}, y_{t-j}$ e $d_{t}$ (variável dummy). A variável aleatória residual, $u_{t}$, procura incluir todas as influências na variável dependente que não podem ser explicadas linearmente pelas variáveis independentes (Brooks, 2008; Wooldridge, 2002).

O método dos mínimos quadrados foi utilizado na estimação dos modelos econométricos. Na prática, foram estimados seis modelos de regressão múltipla com diferentes variáveis explicativas, de acordo com as hipóteses estabelecidas e com o objectivo de comparar as diferenças em termos de ajustamento.

Previamente, foi analisada a estacionariedade das séries temporais de modo a evitar o fenómeno da regressão espúria que ocorre quando as variáveis não estacionárias incluídas no modelo conduzem a um coeficiente de determinação $\left(R^{2}\right)$ elevado, consequência da

\footnotetext{
10 www.acap.pt
} 
existência de uma tendência comum e não de uma relação linear entre elas (Wooldridge, 2002). Realizou-se o teste Augmented Dickey-Fuller (ADF) para detecção de raízes unitárias. A hipótese nula do teste, $H_{0}$, admite a presença de uma raiz unitária. Quando as variáveis não verificam a hipótese de estacionariedade, o teste aplica-se às primeiras diferenças logarítmicas das séries como forma de as estacionarizar (Gujarati, 2003). A Tabela 1 permite concluir que a Procura Interna e o PIB são estacionários na forma logarítmica. Assim, o teste foi aplicado às primeiras diferenças logarítmicas das restantes variáveis, por uma questão de uniformização e interpretação dos resultados. Todas verificam a condição de estacionariedade.

Tabela 1: Resultados do teste Augmented Dickey-Fuller

\begin{tabular}{|c|c|c|c|c|c|c|c|}
\hline Variáveis & LOG_CASFAC & LOG_GAE & LOG_PI & LOG_ACAP & LOG_IMP & LOG_EXP & LOG_PIB \\
\hline $\begin{array}{l}\text { Estatística } \\
\text { do teste ADF }\end{array}$ & $-2,43$ & $-0,85$ & $-3,42$ & $-2,29$ & $-1,56$ & $-1,12$ & $-3,27$ \\
\hline \multicolumn{8}{|l|}{$\begin{array}{l}\text { Valores críticos } \\
\text { do teste }\end{array}$} \\
\hline $1 \%$ & $-3,57$ & $-3,57$ & $-3,57$ & $-3,57$ & $-3,57$ & $-3,57$ & $-3,57$ \\
\hline $5 \%$ & $-2,92$ & $-2,92$ & $-2,92$ & $-2,92$ & $-2,92$ & $-2,92$ & $-2,92$ \\
\hline $10 \%$ & $-2,60$ & $-2,60$ & $-2,60$ & $-2,60$ & $-2,60$ & $-2,60$ & $-2,60$ \\
\hline Probabilidade & 0,14 & 0,79 & 0,01 & 0,18 & 0,50 & 0,70 & 0,02 \\
\hline Variáveis & DLOG_CASFAC & DLOG_GAE & DLOG_PI & DLOG_ACAP & DLOG_IMP & DLOG_EXP & DLOG_PIB \\
\hline $\begin{array}{l}\text { Estatística } \\
\text { do teste ADF }\end{array}$ & $-11,29$ & $-6,38$ & $-6,47$ & $-8,58$ & $-6,51$ & $-6,17$ & $-6,49$ \\
\hline \multicolumn{8}{|l|}{$\begin{array}{l}\text { Valores críticos } \\
\text { do teste }\end{array}$} \\
\hline $1 \%$ & $-3,57$ & $-3,57$ & $-3,57$ & $-3,57$ & $-3,57$ & $-3,57$ & $-3,57$ \\
\hline $5 \%$ & $-2,92$ & $-2,92$ & $-2,92$ & $-2,92$ & $-2,92$ & $-2,92$ & $-2,92$ \\
\hline $10 \%$ & $-2,60$ & $-2,60$ & $-2,60$ & $-2,60$ & $-2,60$ & $-2,60$ & $-2,60$ \\
\hline Probabilidade & 0,00 & 0,00 & 0,00 & 0,00 & 0,00 & 0,00 & 0,00 \\
\hline
\end{tabular}

A verificação de todos os pressupostos do modelo de regressão linear garante que os estimadores obtidos através do método OLS apresentam as propriedades óptimas contidas no teorema de Gauss-Markov.

Seguindo Gujarati (2003), analisou-se a presença de heterocedasticidade através do teste de White. Os modelos apresentaram p-valor que variam entre 0,57 e 0,93 , não se rejeitando a hipótese nula e assegurando-se assim a ausência de heterocedasticidade (Tabela 2).

Para detectar a presença de autocorrelação dos erros procedeu-se à análise do correlograma dos resíduos dos modelos, assim como à realização do teste de Breusch-Godfrey. 
A hipótese nula, $H_{0}$, testa a ausência de autocorrelação dos erros no modelo até quatro desfasamentos ${ }^{11}$. Todos os modelos apresentam ausência de autocorrelação dos erros após a aplicação do teste de Breusch-Godfrey, com resultados entre 0,68 e 0,91.

Tabela 2: Resultados dos testes White, Breusch-Godrey, e Jarque-Bera

\begin{tabular}{|c|c|c|c|c|c|c|}
\hline Modelos & M1 & M2 & M3 & M4 & M5 & M6 \\
\hline \multicolumn{7}{|l|}{ Teste White } \\
\hline F-statistic & 0,67 & 0,33 & 0,45 & 0,43 & 0,79 & 0,71 \\
\hline Prob. $F(8,41)$ & 0,72 & 0,95 & 0,88 & 0,90 & 0,61 & 0,68 \\
\hline$O b s * R$-squared & 5,75 & 3,00 & 4,03 & 3,87 & 6,68 & 6,05 \\
\hline Prob. Chi-Square (8) & 0,68 & 0,93 & 0,85 & 0,87 & 0,57 & 0,64 \\
\hline Scaled explained SS & 5,14 & 3,78 & 4,43 & 3,92 & 6,38 & 6,68 \\
\hline Prob. Chi-Square(8) & 0,74 & 0,88 & 0,82 & 0,86 & 0,60 & 0,57 \\
\hline \multicolumn{7}{|l|}{ Teste Breusch-Godrey } \\
\hline$F$-statistic & 0,30 & 0,47 & 0,51 & 0,21 & 0,21 & 0,37 \\
\hline Prob. F(4,42) & 0,88 & 0,76 & 0,73 & 0,93 & 0,93 & 0,83 \\
\hline Obs*R-squared & 1,38 & 2,15 & 2,32 & 0,97 & 1,00 & 1,70 \\
\hline Prob. Chi-Square(4) & 0,85 & 0,71 & 0,68 & 0,91 & 0,91 & 0,79 \\
\hline \multicolumn{7}{|l|}{ Teste Jarque-Bera } \\
\hline Mean & 0,00 & 0,00 & 0,00 & 0,00 & 0,00 & 0,00 \\
\hline Median & $-0,01$ & 0,00 & 0,00 & 0,00 & $-0,01$ & 0,01 \\
\hline Maximum & 0,17 & 0,11 & 0,14 & 0,13 & 0,18 & 0,13 \\
\hline Minimum & $-0,18$ & $-0,22$ & $-0,21$ & $-0,19$ & $-0,20$ & $-0,23$ \\
\hline Std. Dev & 0,07 & 0,06 & 0,07 & 0,06 & 0,07 & 0,07 \\
\hline Skewness & 0,11 & $-0,60$ & $-0,45$ & $-0,22$ & 0,03 & $-0,46$ \\
\hline Kurtosis & 3,12 & 3,97 & 3,59 & 3,39 & 3,25 & 3,61 \\
\hline farque-Bera & 0,13 & 4,99 & 2,42 & 0,72 & 0,14 & 2,51 \\
\hline Probability & 0,94 & 0,08 & 0,30 & 0,70 & 0,93 & 0,28 \\
\hline
\end{tabular}

Para comprovar a normalidade dos resíduos foi aplicado o teste de Farque-Bera (Greene, 2003). A probabilidade da estatística do teste varia entre 0,08 e 0,94 , não se rejeitando assim a hipótese nula da normalidade dos resíduos. O valor de 0,08 é registado no modelo que

11 Devido à periodicidade das séries de dados utilizadas nos modelos foi escolhido um desfasamento de quatro períodos. Ver Brooks (2008), p.149. 
inclui a Procura Interna, o que apresenta um problema apenas para quando se testa com um nível de significância igual ou superior a $10 \%$.

Para analisar a existência de erros de especificação, utilizou-se o teste Ramsey Reset, o qual tem como objectivo avaliar se o modelo falhou em captar não linearidades importantes. A não rejeição da hipótese nula, $H_{0}$, valida a correcta especificação do modelo. Todos os modelos testados apresentaram valores entre os 0,52 e os 0,93 , encontrando-se correctamente especificados (Tabela 3).

Tabela 3: Resultados do teste Ramsey Reset

\begin{tabular}{lcccccc}
\hline \multicolumn{1}{c}{ Modelos } & M1 & M2 & M3 & M4 & M5 & M6 \\
\hline Teste Ramsey Reset & & & & & & \\
\hline F-statistic (2,44) & 0,07 & 0,66 & 0,39 & 0,57 & 0,22 & 0,15 \\
Likelihood ratio (2) & 0,15 & 1,48 & 0,89 & 1,27 & 0,51 & 0,33 \\
Probability F & 0,94 & 0,52 & 0,68 & 0,57 & 0,80 & 0,86 \\
Probability LR & 0,93 & 0,48 & 0,64 & 0,53 & 0,78 & 0,85 \\
\hline
\end{tabular}

De forma a avaliar se existe um elevado grau de multicolinearidade, foi calculada uma matriz de correlações entre as variáveis independentes para detectar a existência de correlações muito elevadas (Tabela 4). ${ }^{12}$ Foi possível observar que o valor mais elevado se registou entre as Importações e o termo desfasado da concessão de crédito ao consumo, representando $18,42 \%$, concluindo-se que não existe um elevado grau de correlação entre as variáveis.

Outro método utilizado foi o VIF (Variance Inflation Factors), que mede o nível de colinearidade entre os regressores. O método mostra como a variância de um estimador é inflacionada pela presença de multicolinearidade. Caso não exista colinearidade entre duas variáveis, o VIF apresentará o valor de 1 , caso apresente um valor superior a 10 diz-se que é altamente colinear ${ }^{13}$. Observou-se que os valores registados aproximam-se de um, concluindo-se assim que não existe um elevado grau de colineariedade entre as variáveis independentes presentes nos modelos (Tabela 5).

12 Segundo Gujarati (2003, p. 359), caso existam correlações acima dos 0,8 existe grande possibilidade de a multicolinearidade se tornar um problema.

13 Ver Gujarati (2003), p. 351 e p. 362. 
Notas Económicas

Dezembro '17 (65-81)

Tabela 4: Matriz de correlações das variáveis dos modelos

\begin{tabular}{|c|c|c|c|}
\hline \multirow{4}{*}{ M1 } & Variável & DLOG_CASFAC $(-1)$ & $D L O G \_G A E$ \\
\hline & $D L O G \_C A S F A C(-1)$ & 1,00 & 0,13 \\
\hline & $D L O G_{-} G A E$ & 0,13 & 1,00 \\
\hline & $Q 4$ & $-0,16$ & $-0,04$ \\
\hline \multirow{4}{*}{ M2 } & Variável & $D L O G_{-} C A S F A C(-1)$ & $D L O G \_P I$ \\
\hline & $D L O G_{-} C A S F A C(-1)$ & 1,00 & $-0,01$ \\
\hline & $D L O G_{-} P I$ & $-0,01$ & 1,00 \\
\hline & $Q 4$ & $-0,16$ & 0,05 \\
\hline \multirow{4}{*}{ M3 } & Variável & $D L O G_{-} C A S F A C(-1)$ & $D L O G \_A C A P$ \\
\hline & $D L O G_{-} C A S F A C(-1)$ & 1,00 & $-0,11$ \\
\hline & $D L O G \_A C A P$ & $-0,11$ & 1,00 \\
\hline & $Q 4$ & $-0,16$ & 0,09 \\
\hline \multirow{4}{*}{ M4 } & Variável & $D L O G_{-} C A S F A C(-1)$ & $D L O G_{-} I M P$ \\
\hline & $D L O G_{-} C A S F A C(-1)$ & 1,00 & 0,08 \\
\hline & $D L O G \_I M P$ & 0,08 & 1,00 \\
\hline & $Q 4$ & $-0,16$ & 0,01 \\
\hline \multirow{4}{*}{ M5 } & Variável & $D L O G_{-} C A S F A C(-1)$ & $D L O G \_E X P$ \\
\hline & $D L O G_{-} C A S F A C(-1)$ & 1,00 & 0,18 \\
\hline & $D L O G \_E X P$ & 0,18 & 1,00 \\
\hline & $Q 4$ & $-0,16$ & $-0,10$ \\
\hline \multirow{4}{*}{ M6 } & Variável & $D L O G_{-} C A S F A C(-1)$ & $D L O G \_P I B$ \\
\hline & $D L O G_{-} C A S F A C(-1)$ & 1,00 & 0,06 \\
\hline & $D L O G \_P I B$ & 0,06 & 1,00 \\
\hline & $Q 4$ & $-0,16$ & $-0,03$ \\
\hline
\end{tabular}


Tabela 5: Resultados do teste VIF

\begin{tabular}{|c|c|c|c|c|}
\hline & Variável & Coefficient Variance & Uncetered VIF & Centered VIF \\
\hline & $C$ & 0,00 & 1,46 & NA \\
\hline \multirow[t]{5}{*}{ M1 } & $D L O G \_C A S F A C(-1)$ & 0,01 & 1,07 & 1,04 \\
\hline & $D L O G_{-} G A E$ & 0,17 & 1,07 & 1,02 \\
\hline & $Q^{4}$ & 0,00 & 1,39 & 1,03 \\
\hline & Variável & Coefficient Variance & Uncetered VIF & Centered VIF \\
\hline & $C$ & 0,00 & 1,49 & NA \\
\hline \multirow[t]{5}{*}{ M2 } & $D L O G \_C A S F A C(-1)$ & 0,01 & 1,05 & 1,03 \\
\hline & $D L O G_{-} P I$ & 0,81 & 1,09 & 1,00 \\
\hline & Q4 & 0,00 & 1,39 & 1,03 \\
\hline & Variável & Coefficient Variance & Uncetered VIF & Centered VIF \\
\hline & $C$ & 0,00 & 1,42 & NA \\
\hline \multirow[t]{5}{*}{ M3 } & $D L O G_{-} C A S F A C(-1)$ & 0,01 & 1,05 & 1,03 \\
\hline & $D L O G \_A C A P$ & 0,01 & 1,01 & 1,01 \\
\hline & $Q^{4}$ & 0,00 & 1,39 & 1,03 \\
\hline & Variável & Coefficient Variance & Uncetered VIF & Centered VIF \\
\hline & $C$ & 0,00 & 1,47 & NA \\
\hline \multirow[t]{5}{*}{ M4 } & $D L O G \_C A S F A C(-1)$ & 0,01 & 1,06 & 1,03 \\
\hline & $D L O G \_I M P$ & 0,10 & 1,07 & 1,01 \\
\hline & Q4 & 0,00 & 1,39 & 1,03 \\
\hline & Variável & Coefficient Variance & Uncetered VIF & Centered VIF \\
\hline & $C$ & 0,00 & 1,52 & NA \\
\hline \multirow[t]{5}{*}{ M5 } & $D L O G \_C A S F A C(-1)$ & 0,01 & 1,09 & 1,06 \\
\hline & $D L O G \_E X P$ & 0,12 & 1,13 & 1,04 \\
\hline & $Q 4$ & 0,00 & 1,39 & 1,03 \\
\hline & Variável & Coefficient Variance & Uncetered VIF & Centered VIF \\
\hline & $C$ & 0,00 & 1,54 & NA \\
\hline \multirow[t]{3}{*}{ M6 } & $D L O G_{-} C A S F A C(-1)$ & 0,01 & 1,06 & 1,03 \\
\hline & DLOG_PIB & 1,47 & 1,12 & 1,00 \\
\hline & $Q 4$ & 0,00 & 1,39 & 1,03 \\
\hline
\end{tabular}




\section{Resultados}

Analisando os resultados das estimações dos modelos, observa-se que todas as variáveis incluídas são estatisticamente significativas a um nível de significância entre $1 \%$ e $5 \%$ (Tabela 6).

Todos os modelos estimados revelaram-se estatisticamente significativos a um nível de significância de $1 \%$, com um grau de ajustamento entre 40,91\% e 52,23\%.

As variáveis comuns aos modelos estimados são o crédito ao consumo com um desfasamento de um período e uma dummy sazonal, Q4, que representa o $4 .^{\circ}$ trimestre de cada ano do período em análise. É possível concluir que um aumento de $1 \%$ na variação do crédito concedido no período anterior, conduz, em média, a um decréscimo entre $0,41 \%$ e $0,46 \%$ na variação da concessão do crédito corrente, ceteris paribus. Com a inclusão da dummy sazonal, que apresenta coeficientes estatisticamente significativos entre 0,08 e 0,09, para um nível de significância de $1 \%$, confirma-se a presença de sazonalidade.

A hipótese 1 deste estudo é analisada através do modelo 1, no qual se confirma que uma maior abertura da economia ao exterior influencia positivamente a concessão do crédito, sendo que um aumento de $1 \%$ na variação deste indicador resulta em média num acréscimo de $1,43 \%$ na variação do crédito concedido.

A Procura Interna, presente no modelo 2, apresenta um coeficiente de 3,71, sendo a variável com o maior coeficiente estimado, quando comparado com os outros modelos. Conclui-se, perante a hipótese 2 deste estudo, que um aumento do consumo e da capacidade produtiva por parte das empresas ${ }^{14}$ influencia fortemente a concessão de crédito. ${ }^{15}$

Através da análise do modelo 3 é possível confirmar que a venda de automóveis ligeiros influencia a concessão de crédito, apresentando a variável um coeficiente de 0,23, estasticamente significativo a um nível de significância de $1 \%$.

De forma a responder à hipótese 4, e assim analisar a relacão das Importações e Exportações com a concessão de crédito ao consumo, é necessário analisar os modelos 4 e 5 . As Importações revelam ser mais determinantes na concessão de crédito do que as Exportações, apresentando o modelo 4 um grau de ajustamento bastante superior ao do modelo 5 . A variável Importações é estatisticamente significativa a um nível de significância de $1 \%$, apresentando um coeficiente de 1,34. As Exportações apresentam um coeficiente de 0,88, que é estatisticamente significativo a um nível de significância de 5\%.

No modelo 6 foi incluída a variável PIB de forma a serem retiradas conclusões acerca da relação entre a riqueza gerada no país e a concessão de crédito por parte da ASFAC. Observa-se que o PIB apresenta um coeficiente elevado de 3,13, sendo estatisticamente significativo a um nível de significância de $5 \%$.

\footnotetext{
14 Neste caso corresponde à rubrica de Investimento no cálculo da Procura Interna.

15 Consumo efectuado pelos agentes privados e públicos.
} 
Tabela 6: Resultados da estimação dos modelos

\begin{tabular}{|c|c|c|c|c|c|c|}
\hline Variáveis & M 1 & M 2 & M 3 & M 4 & M 5 & M 6 \\
\hline \multirow{2}{*}{ C } & $-0,006$ & $-0,009$ & 0,002 & $-0,008$ & $-0,007$ & $-0,008$ \\
\hline & $(0,012)$ & $(0,012)$ & $(0,012)$ & $(0,011)$ & $(0,013)$ & $(0,013)$ \\
\hline \multirow{2}{*}{ CASFAC (-1) } & $0,457 * * *$ & $0,409 * * *$ & $0,411^{* * *}$ & $0,444^{* * *}$ & $0,459 * * *$ & $0,426^{* * *}$ \\
\hline & $(0,109)$ & $(0,103)$ & $(0,112)$ & $(0,102)$ & $(0,115)$ & $(0,113)$ \\
\hline GAE & $\begin{array}{c}1,432 * * * \\
(0,418)\end{array}$ & - & - & - & - & - \\
\hline$P I$ & - & $\begin{array}{c}3,708^{* * * *} \\
(0,900)\end{array}$ & - & - & - & - \\
\hline VAACAP & - & - & $\begin{array}{c}0,230 * * * \\
(0,081)\end{array}$ & - & - & - \\
\hline$I M P$ & - & - & - & $\begin{array}{c}1,343^{* * * *} \\
(0,310)\end{array}$ & - & - \\
\hline$E X P$ & - & - & - & - & $\begin{array}{c}0,882 * * \\
(0,350)\end{array}$ & - \\
\hline$P I B$ & - & - & - & - & - & $\begin{array}{c}3,129 * * \\
(1,213)\end{array}$ \\
\hline \multirow{2}{*}{$Q^{4}$} & $0,087 * * *$ & $0,081 * * *$ & $0,080^{* * *}$ & $0,084 * * *$ & $0,089 * * *$ & $0,087 * * *$ \\
\hline & $(0,023)$ & $(0,022)$ & $(0,024)$ & $(0,022)$ & $(0,024)$ & $(0,024)$ \\
\hline $\mathrm{R}^{\wedge} 2$ & 0,497 & 0,539 & 0,463 & 0,552 & 0,445 & 0,448 \\
\hline $\operatorname{Adj} R^{\wedge} 2$ & 0,464 & 0,509 & 0,428 & 0,522 & 0,409 & 0,412 \\
\hline $\mathrm{n}$ & 50 & 50 & 50 & 50 & 50 & 50 \\
\hline F-Statistic & $15,138 * * *$ & $17,911^{* * *}$ & $13,238 * * *$ & $19,855^{* * *}$ & $12,309 * * *$ & $12,464^{* * *}$ \\
\hline
\end{tabular}

Notas: *, ** e *** indicam os níveis de significância de 10\%, 5\% e 1\%. O valor entre parêntesis é o desvio-padrão. 


\section{Conalusões}

O crédito total ao consumo tem sido pouco estudado. Este artigo teve como objetivo analisar as variáveis mais relevantes na sua evolução, atendendo à dimensão deste fenómeno na actualidade. Através da análise descritiva concluiu-se que o crédito ao consumo concedido pela ASFAC, entre 1998 e 2011 , revelou uma tendência crescente, com o peso dominante do Crédito Clássico e do Crédito Stock, apesar da forte quebra registada entre 2007 e 2011.

Da análise econométrica concluiu-se que um aumento de $1 \%$ na variação do crédito ao consumo concedido no período anterior, resulta, em média, num decréscimo entre $0,41 \%$ e $0,46 \%$ na variação da concessão do crédito no período corrente. A inclusão do termo autoregressivo desta variável nos modelos foi indispensável para que estes fossem validados nos testes a que foram sujeitos. Foi também identificada a presença de sazonalidade no quarto trimestre. Quando as variáveis macroeconómicas são consideradas como variáveis explicativas, concluiu-se que o modelo que apresenta o melhor grau de ajustamento é o que inclui as Importações, sendo que um aumento de $1 \%$ nas Importações, provoca, em média, um aumento de $1,34 \%$ na variação do crédito concedido.

Para os decisores políticos esta análise é bastante pertinente com vista, por exemplo, a implementação de medidas de fomento da poupança dos particulares. Investigação futura deverá explorar outras variáveis explicativas do comportamento do crédito ao consumo, nomeadamente a competição crescente no mercado de oferta de crédito ao consumo, que poderá explicar o sobrendividamento dos particulares.

\section{REFERÊNGIAS Bibliográfigas}

Attanasio, O.; Weber, G. (2010) Consumption and saving: Models of intertemporal allocation and their implications for public policy, Fournal of Economic Literature, 48(3), 693-751.

Attanasio, O. (1999) Consumption, Handbook of Macroeconomics, in J. Taylor and M. M. Woodford (eds.) Handbook of Macroeconomics, Vol 1, 741-812.

Banco de Portugal (2011) Relatório do Inquérito à Literacia Financeira da População Portuguesa, Lisboa, Banco de Portugal.

Banco de Portugal, Comissão de Mercado de Valores Imobiliários, Instituto de Seguros de Portugal (2012) Plano Nacional de Formação Financeira, Lisboa, BdP, GMVM e ISP.

Banco de Portugal, Comissão de Mercado de Valores Imobiliários, Autoridade de Supervisão de Seguros e Fundos de Pensões (2016) Plano Nacional de Formação Financeira 2016-2020, Lisboa, BdP, GMVM e ASF.

Banks, J.; Blundell, R.; Tanner, S. (1998) Is there a retirement-savings puzzle? American Economic Review, 88 (4), 769-88.

Bertola, G., Disney, R.; Grant, C. (2006) The economics of consumer credit demand and supply, in G. Bertola, R. Disney, and C. Grant (eds.), The Economics of Consumer Credit, Cambridge, MA, MIT Press, 1-26.

BIS (2011) The impact of sovereign credit risk on bank funding conditions, CGFS Papers, No 43, Committee on the Global Financial System, July, Bank for International Settlements.

Brooks, C. (2008) Introductory Econometrics for Finance, New York, Cambridge University Press. 
Browning, T.; Crossley, T. (2001) The life-cycle model of consumption and saving, fournal of Economic Perspectives, 15 (3), 3-22.

Campbell, J. (2006) Household finance, Fournal of Finance, 61 (4), 1553-1604.

Comissão Europeia (2008) Towards a Common Operational European Definition of Overindebtedness, Bruxelas, Comissão Europeia.

Costa, S.; Farinha, L. (2012) O endividamento das famílias: Uma análise microeconómica com base nos resultados do inquérito à situação financeira das famílias, in Relatório de Estabilidade Financeira, Banco de Portugal, pp. 137-164.

Coudert, V. (1990) Les disparités internationales de comportements d'épargne: Une analyse des EtatsUnis, du Japon et de l'Europe par le cycle de vie, Économie et Statistic, 232, 49-67.

Deaton, A. (2005) Franco Modigliani and the life-cycle theory of consumption, BNL Quarterly Review, Banca Nazionale del Lavorno, 58, 91-107.

Dick, A.; Lehnert, A. (2010) Personal bankruptcy and credit market competition, Fournal of Finance, 65(2), 655-686.

Fay, S.; Hurst, E.; White, M. (2002) The household bankruptcy decision, American Economic Review, 92(3), 706-718.

Friedman, M. (1957) The Permanent Income Hypothesis, in M. Friedman (ed.), A Theory of the Consumption Function, Princeton, Princeton University Press, 20-37.

Garcia, M. T. M. (2013) A Poupança e os Sistemas de Pensões, Colecção Económicas (2 ${ }^{\mathrm{a}}$ Série), Coimbra, Almediana.

Greene, W. (2003) Econometric Analysis, New Jersey, Prentice Hall.

Gujarati, D. (2003) Basic Econometrics, New York, McGraw-Hill.

Himmelstein, D.; Thorne, D.; Warren, E.; Woolhandler, S. (2009) Medical bankruptcy in the United States, American Fournal of Medicine, 122 (8), 741-74.

Masson, A. (1991) La théorie du cycle de vie illlustrée, Risques, 7, 75-97.

Meghir, C. (2004) A retrospective on Friedman's theory of permanent income, Economic Fournal, 114, F293-F306.

Modigliani, F. (1988) The role of intergenerational transfers and life cycle saving in the accumulation of wealth, Fournal of Economic Perspectives, 2 (2), 15-40.

Modigliani, F.; Brumberg, R. (1954) Utility Analysis and the Consumption Function, An Interpretation of Cross-Section Data, in K. Kurihara (ed.), Post-Keynesian Economics, New Brunswick, Rutgers University Press, pp. 388-436.

Rocher, S.; Stierle, M. H. (2015) Household saving rates in the EU: Why do they differ so much? Discussion Paper 005, European Economy, European Commission Directorate-General for Economic and Financial Affairs.

Santos, A. C., Costa, V.; Teles, N. (2013) A economia política do consumo e do crédito às famílias: Um contributo interdisciplinar, Revista Crítica de Ciências Sociais, 101, 9-38.

Sullivan, T.; Warren, E., Westbrook, J. (2000) The Fragile Middle Class: American in Debt, New Haven, Yale University Press, pp. 1-26.

Wooldridge, J. (2002) Introductory Econometrics: A Modern Approach, Mason, Thomson South-Western. 